\title{
An Implementation of Decision Making Models and Mechanisms for Multi-Agent Systems in Competitive Environments
}

\author{
Wei Wu, Behrouz Homayoun Far, Armin Eberlein \\ Department of Electrical and Computer Engineering \\ University of Calgary \\ 2500, University Drive, N.W. \\ Calgary, Alberta, Canada, T2N 1N4 \\ \{wuwe, far, eberlein\}@enel.ucalgary.ca
}

\begin{abstract}
In Multi-Agent System (MAS) competitive environment, agents' reasoning process is more complex due to the fact that uncertainty factor is involved. Decision making models and mechanisms for MAS are studied in this paper from two viewpoints: $S-R-K$ human information processing model viewpoint, and game theory and decision theory viewpoint. Five decision making modules that are rule-based module, Dempster Shafer module, certainty module, risk module and uncertainty module are developed and the reasoning procedures are discussed. These modular processing techniques could improve the quality and performance of MAS design and development.
\end{abstract}

Keywords: Multi-Agent System, competition, reasoning engine.

\section{INTRODUCTION}

Multi-Agent Systems are considered as a new experimental embodiment of computer programs. In such systems, the agents are autonomous and typically heterogeneous. Heterogeneous agents' interactions, i.e., cooperation, coordination and competition, are of special interest because knowledge, action and control are distributed among agents. Research in this paper is concerned with the reasoning process of intelligent agents in competitive environments. Competition relationship among agents could be divided in to two types [2]: loose competition and strict competition. Loose competition means that an agent's goals are revealed but the knowledge behind it is masked from the other parties, strict competition means that neither an agent's goals nor the knowledge behind it is revealed to the other parties. In competitive environments, an agent may try to hide its strategy and the knowledge behind it as much as possible, therefore agents should use uncertain data to reason.
In this paper, models and mechanisms for strategic decision making for MAS in competitive environments are studied and a modular implementation of the reasoning engine is presented. In section 2 we will look at the reasoning procedures inside an agent which are based on the Skills, Rules and Knowledge (S-R-K) taxonomy [4], and analyze the relationship between an agent and its opponents based on game theory and decision theory, from which, a decision-making model consists of five modules is developed. The specifications of five decisionmaking modules are illustrated in section 3 and their benefits are concluded at section 4 .

\section{METHODOLOGY}

\subsection{S-R-K Hierarchical Reasoning Procedure}

Rasmussen's S-R-K human information processing model $[3,4]$ is adopted to reduce the complexity of agents' reasoning process. In the S-R-K perspective [3,4], the skill-based level denotes almost routines performances without conscious attention or control. At this level, an agent's behavior is governed by stored patterns of predefined procedures in its own knowledge base. The rule-based level represents more conscious behavior when handling familiar situations in which the agent's action is consciously controlled by a set of stored rules or procedure that may have derived empirically during previous occasions. The knowledge-based level accounts for unfamiliar situations for which know-how or rules are not available, in this case, the control of reasoning must move to a higher conceptual level, in which behavior is controlled by goals, utilities and the belief about the other parties which we will examine at Section 3.

As illustrated in Fig. 1, three types of reasoning procedures: reactive, planning and decision making process could be adopted by an agent in competitive 
environments in order to take into account different situations which arise in MSA: routine, familiar and unfamiliar situations [1]. The complexity of process is reduced from decision-making process to reactive one, and reactive process dealing with routine tasks needs the least time and effort.

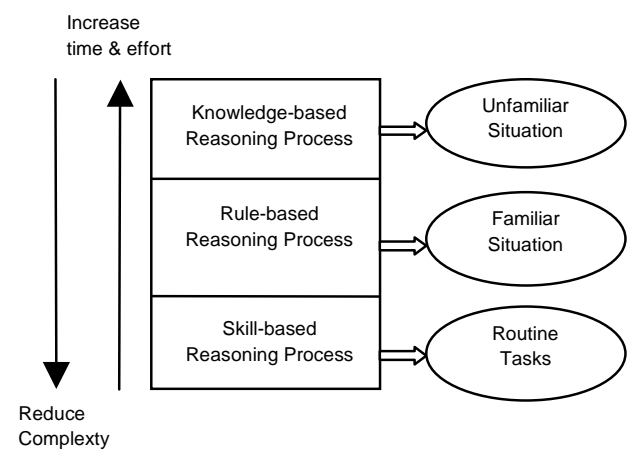

Figure 1. Hierarchical representation of an agent's reasoning process based on the S-R-K model

\subsection{Relationship between Agent and its Opponents}

The relationship between an agent and its opponents means the degree to which an agent knows the goals, preference relationships and strategies of other agents. Game theory [5] and decision theory provide theoretical foundations for modeling different levels of uncertainty and developing agents' decision making mechanisms in competitive environments. An agent's reasoning process could be divided into the following three levels according to the amount of information it gets from the other parties and the environment [2].

- Decision Making under Certainty

An agent knows exactly the state of nature. Therefore, selecting a strategy is straightforward: it selects the strategy which could maximize its utility function [2].

- Decision Making under Risk

An agent is not sure what state of nature will be but it has a probability distribution over the state of nature. In this case, the agent uses the probability distribution as its belief and then selects its own strategy in order to get the maximum utility. A variant named attitude toward risk is also adopted to deal with risk [2].

\section{- Decision Making under Uncertainty}

An agent does not know the state of nature. It uses the degree of optimism quantified by Ordered Weighted Averaging (OWA) operator [6] as its belief to select its own strategy.

Based on the S-R-K model and the relationship between an agent and its opponents, a modular representation of an agent's reasoning process (Fig. 2) is developed and includes five decision making modules: rule-based module, Dempster Shafer module, certainty module, risk module and uncertainty module.

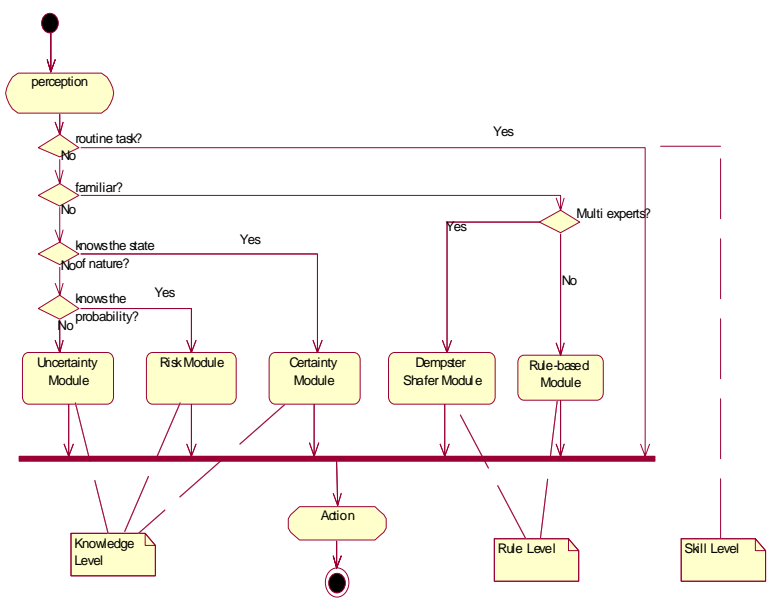

Figure 2. Modular representation of agent's reasoning process

From the reasoning phases (described below) we could easily find that each module can address a specific class of situations. First the information is perceived from the environment, the information which is considered as routine task leads the agent to execute an action directly. If the information is a familiar situation instead of routine task, the agent tries to recognize the information in terms of multi experts or single expert which means that whether it has several alternatives to select to commit an action. In case of single expert, rule-based module is invoked, otherwise, Dempster-Shafer module is invoked. If the information is considered as an unfamiliar situation, three modules could be invoked for different levels of uncertainty of the perceived information. Certainty module manipulates decision-making tasks when the state of nature is known; risk module deals with decisionmaking procedures when the state of nature is unknown but the probability distribution over the state of nature is known; uncertainty module takes care of decision-making procedures when neither state of nature nor the probability distribution is known. Apparently, rule-based module and Dempster Shafer module are at rule-based level while certainty module, risk module and uncertainty module are at knowledge-based level based of the S-R-K model. No module is located at skill-based level because the behavior at this level is a result of reflexive actions rather than decision making process in which a selection from possible choices is to be made. 


\section{SPECIFICATION}

\subsection{Rule-based Module}

In this module, the agent could get suggested solutions that could be considered as rules from its own knowledge base, or from other consultant agents if it is in a MAS. It retrieves appropriate rules from its knowledge base or its communication engine, modifies the adapted plan, and then uses this plan for taking action. Finally, it stores the new rule in its knowledge base for future use.

\subsection{Dempster Shafer Module}

In this module, the agent can get several alternative solutions that should be evaluated and weighted in order to find the best solution. We use Dempster-Shafer theory [7] to achieve this goal.

Based on Dempster-Shafer theory, the alternative solutions are considered as some events $q_{1}, q_{2}, \ldots, q_{n}$, and a set of mutually exclusive possibilities that is called Frame of Discernment $\mathbf{F}$ is generated. $\mathbf{F}$ is the power set, $2^{\theta}$, of the set of all possible conclusions, that is, $\left(\Phi,\left\{q_{1}\right\},\left\{q_{2}\right\},\left\{q_{3}\right\}, \ldots,\left\{q_{n}\right\},\left\{q_{1} q_{2}\right\},\left\{q_{1} q_{3}\right\}, \ldots,\left\{q_{1} q_{n}\right\},\left\{q_{2} q_{3}\right\}\right.$, $\left.\ldots,\left\{q_{2} q_{n}\right\}, \ldots,\left\{q_{1} q_{2} q_{3}\right\}, \ldots,\left\{q_{1} q_{2} q_{n}\right\}, \ldots,\left\{q_{1} q_{2} q_{3} \ldots q_{n}\right\}\right)$ $\mathbf{F}$ is sent to consultant agents. A mass probability function, $\boldsymbol{m}$, which is a numeric value from the range $[0,1]$ to every item in the set $\mathbf{F}$, is assigned by every consultant agent. The belief, Bel, of each element in the set $\mathbf{F}$ is calculated to denote the likelihood that one of its members is the conclusion. In addition, the belief interval [Bel, 1-Bel] is generated for each element in the set $\mathbf{F}$ to express the range of certainty about the belief that the conclusion in the subset of $\mathbf{F}$ is true.

In MAS, it is possible to have more than one consultant agents, so several mass probabilities may be gotten in this module, if we use $\boldsymbol{m}_{1}$ and $\boldsymbol{m}_{2}$ to represent the first and second set of mass probability respectively, the combined mass probabilities could be derived from the following Dempster's combination rule,

$$
m_{3}(C)=\frac{\sum_{X \cap Y=C} m_{1}(X) \times m_{2}(Y)}{1-\sum_{X \cap Y=\phi} m_{1}(X) \times m_{2}(Y)}
$$

Using this combination rule alternatively, we could get the combined mass probabilities over each set of $\mathbf{F}$ of several alternatives that are provided by all consultant agents in its MAS. The best fitting conclusion is selected according to the combined mass probabilities and belief intervals for committing an action.

\subsection{Certainty Module}

Certainty, risk and uncertainty modules are based on the game theory and decision theory which provide a powerful tool and a set of mathematical techniques for making decisions about what action to take when the outcomes of the various actions are not certain. In our study, agents are considered as canonical examples of the rational decision makers that follow these two theories, therefore, agents competitive interactions could be classified into strategic games and extensive games, the former is a model of a situation in which each agent chooses its plan of action once and for all, and all agents' decisions are made simultaneously, by contract, the later specifies a kind of situation that each agent can consider its plan of action not only at the beginning of the game but also whenever he has to make a decision [5]. Within these two classes of games, other situations also exist which model the amount of information (an agent knows the characteristic or previous actions at each action point of the other parties or not). We use the certainty module to model games with perfect information, and risk module to represent games with imperfect information.

\section{- $\quad$ Strategic games in certainty module}

According to game theory [5], a strategic game is modeled as: $\left\{\mathrm{N}, \mathrm{A}_{i}, \geq_{i}\right\}$, where, $\mathrm{N}$ defines the set of agents, $A_{\mathrm{i}}$ is the set of actions available to agent $i, \geq_{i}$ is a preference relation of agent $i$, under a wide range of circumstances the preference relation can be represented by a utility function $\mathrm{u}_{i}$.

A suggested reasonable solution concept called Nash equilibrium is also defined as a profile $\mathrm{a}^{*} \in \mathrm{A}$ of actions with the property that for every player $\mathrm{i} \in \mathrm{N}$ we have

$$
\left(a_{-i}^{*}, a_{i}^{*}\right) \geq_{i}\left(a_{-i}^{*}, a_{i}\right) \text { for all } a_{i} \in A_{i}
$$

\section{- Extensive games in certainty module}

An extensive game with perfect information is modeled as $\left\{\mathrm{N}, \mathrm{H}, \mathrm{P}, \geq_{i}\right\}$, where, similar to strategic game, $\mathrm{N}$ is the set of agents, $\mathrm{H}$ is a set of sequences (finite or infinite), $\mathrm{P}$ is the agent function that assigns to each nonterminal history a member of $\mathrm{N}$, and $\geq_{i}$ is a preference relation of agent $i$.

The reasonable solution profile for extensive game with perfect information named Subgame Perfect Equilibrium is defined to be a strategy profile $\mathrm{s}^{*}$ for which for any history $h$ the strategy profile $\left.s^{*}\right|_{h}$ is a Nash equilibrium of the subgame.

Based on the above definition, the process of the certainty module is first using game theory model to specify the interaction environment and then producing a set of strategy profile based on the associated equilibrium. 


\subsection{Risk Module}

Risk module models agents' interaction in terms of the strategic and extensive games with imperfect information. When an agent calculates its utility functions, its attitude towards risk. How the probability of winning influences decision making is also reflected in this module [2].

- Strategic games in risk module

A strategic game with imperfect information is defined as $\left\{\mathrm{N}, \Omega, A_{i}, \mathrm{~T}_{i}, \tau_{i}, \mathrm{p}_{i}, \geq_{i}\right\}$, where, $\mathrm{N}$ is the set of agents, $\Omega$ is a set of states, $A_{i}$ is a set of actions available to agent $i, \mathrm{~T}_{i}$ is a set of signals that may be observed by agent $i, \tau_{i}: \Omega \rightarrow \mathrm{T}_{i}$ is the signal function of agent $i, \mathrm{p}_{i}$ is the prior belief of agent $i$, and finally $\geq_{i}$ is a preference relation of agent $i$.

Therefore, the associated solution concept called Bayesian Nash Equilibrium is that each agent chooses the best action available to it given the signal that it receives and its belief about the state and the other agents' actions that it deduces from this signal [5].

- Extensive games in risk module

The formal definition of this family of games is $\{\mathrm{N}$, $\left.\mathrm{H}, \mathrm{P}, \Theta_{i}, \mathrm{p}_{i}, \mathrm{u}_{i}\right\}$, where, the meaning of $\mathrm{N}, \mathrm{H}, \mathrm{P}$ is the same as the extensive game in certainty module, $\Theta_{i}$ is a set of possible types of agent $i, \mathrm{p}_{i}$ is the probability measurement on $\Theta_{i}, u_{i}$ is the utility function.

The suggested solution concept for this game family called Perfect Bayesian Equilibrium is a pair $\left(\left(\sigma_{i}\right),\left(\mu_{i}\right)\right)=\left(\left(\sigma_{i}\left(\theta_{i}\right)\right)_{i \in N, \theta_{i} \in \Theta_{i}},\left(\mu_{i}(h)\right)_{i \in N, h \in H}\right)$ where $\sigma_{i}\left(\theta_{i}\right)$ is a behavioral strategy of agent $i$ in $\{\mathrm{N}, \mathrm{H}, \mathrm{P}\}$ and $\mu_{i}(h)$ is a probability measure on $\Theta_{i}$ if it satisfied these four conditions: sequential rationality, correct initial beliefs, action-determined beliefs and Bayesian updating [5].

Based on the above argument, the procedure inside risk module is as follows: first, the agent calculates the utility function with consideration of the agents' attitude towards risk; second, it defines the interactions according to different game models; finally, it gets the reasonable solution profile based on associated equilibrium.

\subsection{Uncertainty Module}

This module is developed for the family of agents' interaction in which the state of nature is unknown. In this case, an agent has to assign its belief without using a probability distribution [2]. We adopt a concept of degree of optimism and using OWA operators treat weight values as pseudo probability distribution and assign it as its belief. Using OWA operator, the degree of optimism is defined as follows [2],

$$
\operatorname{Opt}(W)=\sum_{j} w_{j}(n-j) /(n-1)
$$

Using this definition, an agent could subjectively decide its degree of optimism $O p t(W)$ and input this value into the following linear programming equation,

$$
\max \quad-\sum_{j} w_{j} \log _{2} w_{j}
$$

Subject to:

$$
\begin{aligned}
& \operatorname{Opt}(W)=\sum_{j} w_{j}(n-j) /(n-1) \\
& \operatorname{Opt}(W) \in[0,1] \\
& \sum_{j} w_{j}=1 \\
& w_{j} \geq 0 \text { for } j=1, \ldots, n
\end{aligned}
$$

Then a set of weight values are calculated and could be treated as pseudo probabilities. Once the probabilities are known, we can view decision making process in uncertainty module the same as in the risk module.

\section{CONCLUSION}

In this paper, we analyzed intelligent agents' reasoning process in competitive MAS environments. Based on S$\mathrm{R}-\mathrm{K}$ information processing model, agents' reasoning process is defined at three levels: Skill-based, Rule-based and Knowledge-based. By defining these three levels appropriately, the complexity of the reasoning process of agents could be reduced both at the Skill and Rule-based levels. At knowledge-based level, we use game theory and decision theory to specify agents' interactions and get the reasonable solutions according to the associated equilibriums. We also suggested an implementation for proposed processes. This could enhance the reliability of the agents' reasoning process which is a crucial factor at MAS design.

\section{References}

[1] B.Chaib-draa. Interaction between Agents in Routine, Familiar and Unfamiliar Situations. International Journal of Intelligent \& Cooperative information System, Vol 5(1), pp. 1-25, 1996.

[2] Hiroyuki ONJO, Behrouz H. Far. A Unified View of Heterogeneous Agents' Interaction, IEICE TRANS. INF. \& SYST., VOL. E84-C, No. 8, 2001.

[3] J. Rasmussen. The Role of Hierarchical Knowledge Representation in Decisionmaking and System Management. IEEE TRANS. SYS.MAN \& CYBE, Vol. SMC-15, NO.2, 1985.

[4] J. Rasmussen. Information Processing and Human-Machine Interaction: An Approach to Cognitive Engineering, North Holland. 1986.

[5] M. J. Osborne, A. Rubinstein. A Course in Game Theory. The MIT Press. Cambridge, Massachusetts. London, England. 1996.

[6] P.R. Yager. On Ordered Weighted Averaging Aggregation Operators in Multi-criteria Decision Making.” IEEE TRANS. SMC, No. 18, 1988.

[7] Avelino J. Gonzalez \& Douglas D. Dankel. The Engineering of Knowledge-based Systems: Theory and Practice. Prentice Hall. 